\title{
Pattern recognition in bees
}

\section{Howard C. Howland*}

Department of Neurobiology and Behavior, Cornell University, Ithaca, NY, USA

*Correspondence: hch2@cornell.edu

\section{A book review on \\ What does the honey bee see, and how do we know? a critique of scientific reason}

by Adrian Horridge, Australian National University E-Press (Open Access), 2009, xxiv+360 pages, 113 figures and 575 references. ISBN: $9781921536651 \mathrm{pdf}$

Adrian Horridge has written an interesting, useful, and very opinionated book on the visual system of insects. He states that it could be used by university students interested in the physiology of vision or the psychology of perception, by entomologists or zoologists, by engineers interested in robot vision, and by philosophers interested in the scientific method.

The book is actually several small books in one. It is a history of neuroethological research on insect vision, a functional anatomy of insect visual systems, a compilation of his own studies on pattern vision of bees, and a critique of the scientific reasoning of other workers in the field. The author deserves great praise for publishing the book in an open access mode. While hardcopy books may be purchased, it is freely available in pdf format on the web at http://epress.anu.edu.au/honeybee_citation.html.

Unfortunately, his critique of fellow workers in this field is often harsh, and his dismissal of their work is frequently written, as the post-modernist historians would say, "from a presentist point of view". It is also often both eccentric and extreme. For example: "In a topic such as vision, an analytical mathematical theory is not appropriate" ( $p$. $\mathrm{XV}$ ). "The second corrosive influence (the first was gestalt psychology) was the development of ethology by Konrad Lorenz and Niko Tinbergen, who used intuitive concepts such as 'fear' and 'drive"” (p. 31). "The whole subject (of bee's visual discrimination) was dominated by German biologists who somehow thought that empiricism was immoral" (p. 32). "In the case of vision, the exact relation between input and output is even less useful. In the bee, these searches for exact relations between the features of the pattern and the percentage of correct responses hampered progress.” (p. 33).

I have cited a few of these dismissive statements now as a warning to the reader. If one manages to ignore such comments, there is much to be learned from the book.

The book has 12 chapters for which Horridge provides summaries. Briefly they are: Chapter 1. Early German work, 2. A discourse on scientific method, 3. German work from 1950 on. 4. Work of von Frisch and other behaviorists, 5. and 6. Anatomy and physiology of insects' visual systems. 7. Australian work on the visual control of flight, 8. Von Frisch's work on navigation. 9. Work in Horridge's laboratory on feature detectors. 10. Theory of bee goal recognition. 11. and 12. On whether or not bees see shapes. These chapters are followed by two other sections labeled "Afterthoughts" and "Summary of the model of the bees' visual processing". A short summary of the principal arguments of his theory of bee pattern recognition and some of the historical work underlying it is provided in a recent review (Horridge, 2009).

In this review I have concentrated on Horridge's own researches and the history leading up to them in chapters $1,3,4,9,10$ and 11 . To understand the main thrust of this research, it is best to start at the end with the author's final summary model. This is because most the rest of the book may be seen as a defense of this model, the discovery of the information needed to support it, and a critique of all other research that did not lead to it.

Briefly, Horridge's theory states that the bee eye consists of a very large number of small groups of seven omnitidia that act in concert as various kinds of feature, or "cue" detectors. He distinguishes two kinds of fea- ture detectors: phasic modulation detectors that detect heterochromatic, temporal modulation and tonic detectors of edge orientation. A number of these small groups form a "local area" of 15 to 30 degrees in width, and "Within each region no more than one cue of each kind [presumably phasic or tonic] is detected" (p. 317). Horridge states that there are few kinds of cues, and he lists the order of their preference for learning: “...total modulation in a local area (is) the most preferred, then area, position of a centre, a black spot, colour, radial edges, bilateral symmetry, average orientation and finally tangential or circular edges, which (latter) are avoided." (p. 314). Groups of cues detected at the same time form the "label" of a landmark, and this label can be learned. Such cues are retinotopic and a group of landmark labels at wide angles to each other makes "the key to the recognition of place". It will be seen that this division into small angle and wide angle visual fields is important in understanding the apparent contradictions in experimental results.

The data for Horridge's model were obtained using a behavioral paradigm that was initiated by Hertz (1929), whose work Horridge reviews. In her experiments, bees were shown various pairs of black and white patterns on a horizontal surface, and were rewarded for going to one of these patterns. Then the reward was removed and either the pattern to which the bee was trained, or the unrewarded pattern was exchanged for a pattern of similar but not identical characteristics. She then observed whether or not the bees' visiting behavior changed. The orientation of the table relative to the surrounding landmarks was varied at random. Hertz realized that there might be an untrained attractiveness 
or repulsiveness to a particular pattern, and found this in the relative ease of training bees to such forms. She also discovered: (1) that bees distinguished between strongly branched or entire patterns (e.g. between a cross and a solid circle) (2) That among the solid patterns they distinguished between those with smooth and undulating contours (e.g. a solid circle and a cog-wheel) (3) Among the branched patterns they distinguished between those with many and few branches. Hertz emphasized the fact that, as there was no fixation point, the bees could approach the patterns from any direction, and thus there was no possibility that the bees could use a particular retinal location for analyzing the sensory data (Hertz, 1929, p. 698). Thus her early experiments differed from Horridge's and others' experiments where the bees could only view vertical patterns from a small range of fixed distances in a two choice maze, and where the hole leading to the reward always formed a fixation point.

Hertz concluded that, in agreement with gestalt theory, the forms were distinguished as totalities according to the relationships of their sensory data, and that a "form sense" was conclusively proven ("zwingend erwiesen”) (Hertz, 1929, p. 731). Horridge criticizes Hertz for classifying patterns “...by arbitrary global characters such as symmetry, disruption, isotropy, smoothness, texture, variability of patch size, and separation into parts", noting that "There was, however, no demonstration that these categories really had any meaning for the bees (p. 10)." This criticism is difficult to understand when Hertz's classification is compared with Horridge's listing of preferred cues above. Since both Hertz and Horridge have shown that bees respond to these cues, it must be that Horridge is using the word "meaning" above to refer to the lack of some process in the bee's brain that he associates with Hertz's gestalt hypothesis. And with this realization we come to the crux of Horridge's problems with scientific reasoning.

On the one hand, despite the fact that the real data for his theory of pattern recognition is based on behavioral experiments alone, he does not seem to appreciate the limitation of his behavioral methods to differentiate among the myriad of ways such choice behavior could be instantiated in the nervous system. One of the possible ways, that behavioral experiments have neither confirmed nor falsified, is that the bee's learning process stores and classifies groups of visual cues associated with training patterns, and compares the incoming sensory data with these classified groups. But whether or not the bee used such a scheme, the question of "meaning" within the brain of the insect would appear to be unanswerable, along with other questions of qualia.

The assumption of the identity of the qualia of individual humans, based on their descriptions of them, underlies and provides support for gestalt theory. Hertz applied gestalt theory to insects where such description does not exist and where, given the great evolutionary distance between humans and insects, any assumption about qualia or meaning seems unwarranted. However, what Horridge does not seem to appreciate is that it was the impetus from gestalt theory that gave rise to Hertz's fundamental experiments that have served as the paradigm for the field ever since.

The experimental method of Hertz has been modified and refined over time. Hertz's experiments were performed on a horizontal table and the patterns were rotated at random. Horridge remarks that "...the bees did not correlate edge orientations with their sun compass as they flew in all directions over them." However he misses the point that they could not have correlated them with their sun compass because the table on which the patterns were displayed was rotated at random during the experiments.

Horridge briefly reviews Rudiger Wehner's work which figured prominently in subsequent investigations of bee pattern recognition. In 1968 Wehner introduced a transparent screen between the targets and the bee, which could only approach the reward through a tunnel. This insured that there was a minimum distance from which the bee could view the target, which, in this apparatus provided a $130^{\circ}$ view of the target. Subsequently in Horridge's laboratory the minimum and maximum distances were also controlled using a "Y" maze with an antechamber in front of the transparent partition (Figure 1.1, p. 6). His apparatus provided a $27^{\circ}$ view of the target. Wehner using wide-angle cross and parallel line targets found that the bees' responses to targets which differed by $4^{\circ}$ in orientation were significantly different. However, using narrower angle targets of $42^{\circ}$, Srinivasan et al. (1994) also showed that bees could not distinguish between an upright cross and the same cross rotated by $45^{\circ}$. Srinivasan explained this result by assuming that the crosses were detected by orientation channels that had half widths of $90^{\circ}$. These experiments revealed the essential difference between large angle and narrow angle vision in the bee.

Horridge, having rejected Hertz's gestalt theories as anthropomorphism, does not hesitate to criticize his colleagues when they stray in the direction of gestalt theories of visual recognition. Chen et al. (2003) conducted a number of experiments to test the theory that bees could distinguish between the topological properties of closed and open figures, for example between O's and S's. They demonstrated that bees, trained with rewarded closed $O$ patterns paired with unrewarded open $S$ patterns, transferred this learning to pairs of closed squares vs. open X's, and to closed O's vs. solid circles. as well as open O's vs. otherwise solid circles with four open holes. However they did not discriminate between closed $\mathrm{O}$ and closed square patterns. Chen et al. also showed that bees could be trained to positive $S$ patterns and that they could distinguish between closed $\theta$ patterns against $S$ patterns even where the test symbols were rotated $45^{\circ}$ from the training position. They also showed that the topologically similar patterns had very different two-dimensional Fourier spectra, while topologically different patterns had very similar Fourier spectra.

In a set of ingenious experiments Horridge showed that the $\mathrm{O}$ vs. S discrimination could be explained by simple avoidance cues, namely black near the reward hole (always in the center of the patterns) and an edge orientation at a certain position. However this explanation could not explain why Chen et al's. results that, after training on rewarded O's and unrewarded S's, the bees significantly favored $\mathrm{O}$ vs. a solid circle, $\mathrm{O}$ vs. a square, or a $\theta$ vs. an $S$, both rotated $45^{\circ}$ to the left. Thus Horridge's theory could explain many, but not all of Chen et al's results.

It will be noted that Horridge's experimental apparatus limited the testing of behavior to two alternative forced choices of vertical targets viewed through narrow angles (mostly $30^{\circ}$ or less). He has little to 
say about the behavior of bees that distinguish between targets presented at larger angles, or targets that are not vertical, or that are presented at varying distances $\mathrm{He}$ simply describes targets viewed at large angles as "place recognition" or the learning of multiple landmarks and leaves it at that. (For a recent experiment using wide angle (up to $67^{\circ}$ ) targets that explores the discrimination and categorization of facelike stimuli in honeybees the reader may consult Avarguès-Weber et al., 2010).

Elsewhere in his book (pp. 55-59), Horridge criticizes Reichardt and his colleagues for studying the optomotor reflex of insects when the insect's head is tethered. However, he seems unaware of the fact that his own work has progressed by making just this sort of simplification, forcing the bees to view only narrow angle targets from a fixed distance, and thus ignoring many aspects of the bee's natural behavior.
All in all, there is no question that Adrian Horridge has made very significant contributions to the understanding of pattern recognition in bees, and that he has constructed a very plausible model of their behavior to small angle targets. His book is an excellent introduction to the subject, despite, or even sometimes because, of his very opinionated outlook. I say "despite", because if one would believe him, the behavioral side of the studies are virtually finished, and they definitely are not. But also "because", since a careful reading of the book will reveal the many interesting experiments of wide angle vision that remain to be done, along with the impetus that the reader is continually challenged to prove some of his most provocative statements wrong.

\section{REFERENCES}

Avarguès-Weber, A., Portelli, G. Benard, J., Dyer, A., and Giurfa, M. (2010). Configural processing enables discrimination and categorization of face-like stimuli in honeybees. J. Exp. Biol. 213, 593-601.

Chen, L., Zhang, S., and Srinivasan, M. V. (2003). Global perception in small grains: topological recognition in honey bees. PNAS 100, 6884-6889.

Hertz, M. (1929). Die Organisation des optischen Feldes bei der Biene I. Z. Vgl. Physiol. 8, 593-748.

Horridge, A. (2009). Commentary: what does an insect see. J. Exp. Biol. 212, 2721-2729.

Srinivasan, M. V., Zhang, W. W., and Witne, K. (1994). Visual discrimination of pattern orientation by honeybees: performance and implications for 'cortical processing'. Phil. Trans. R. Soc. Lond. B 343, 199-210.

Received: 27 February 2010; accepted: 04 March 2010; published online: 17 March 2010.

Citation: Howland HC (2010) Pattern recognition in bees. Front. Behav. Neurosci. 4:11. doi: 10.3389/fnbeh.2010.00011

Copyright $\odot 2010$ Howland. This is an open-access article subject to an exclusive license agreement between the authors and the Frontiers Research Foundation, which permits unrestricted use, distribution, and reproduction in any medium, provided the original authors and source are credited. 